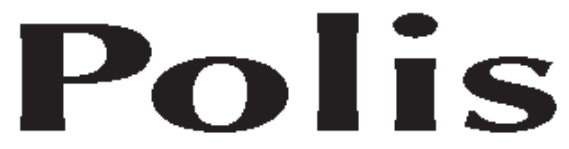

\title{
El conflicto entre eficacia y sostenibilidad: Utilizar el "capital mineral" de la Tierra o el flujo solar y sus derivados renovables*
}

Resumen: Se aborda aquí el problema del petróleo desde un enfoque más amplio y clarificador que permite visualizar la verdadera encrucijada en que se encuentra la civilización industrial. Se propone en este artículo una metodología para el cálculo del coste físico en términos energéticos del coste de reposición del capital mineral del planeta y como criterio para determinar la sustentabilidad de la explotación de un recurso mineral hasta el cierre del ciclo de materiales, considerando así los costes de extracción y reposición.

Palabras clave: crecimiento exponencial, ciclo de materiales, agotamiento del petróleo

\section{The conflict between efficacy and sustainability: Utilize the «mineral capital» of the Earth or the solar flow and its renewable by-products}

Abstract: The problem of petroleum is undertaken here in a more extensive and clarifying scope that allows us to visualize the true crossroads which the industrial civilization is confronting. A methodology is proposed in this article for calculation of the physical price in terms of energy of the price of reinstatement of the mineral capital of the planet, and as criterion to determine the sustainability of the exploitation of a mineral resource up to the closing of the cycle of materials, considering thus the prices of extraction and reinstatement.

Key words: exponential growth, cycle of materials, exhaustion of petroleum

Recibido: 01.09.08

Aceptado: 25.09.08

$$
* * *
$$

\footnotetext{
* Este texto reproduce, con algunas actualizaciones, el publicado en el libro Sempere J. y Tello, E. (coords.) (2007) El final de la era del petróleo barato, Barcelona, Icaria (pp.185-199).

${ }^{* *}$ Universidad Politécnica de Madrid, España. Email: jmn@telefonica.net
} 


\section{Introducción: sobre el "agotamiento" del petróleo... y de los otros minerales de la corteza terrestre.}

El problema del agotamiento del petróleo fue una preocupación generalizada en la década de los setenta. Las "crisis petrolíferas" de 1973 y 1979, motivadas por la elevación de los precios del crudo promovidas por los países de la OPEP, propiciaron este tipo de reflexiones que llevaron a vaticinar que la civilización industrial colapsaría sobre todo por escasez de recursos. Sin embargo, treinta años después, cuando el consumo global de energía ha amentado en más de un $70 \%$ y cuando hay mucho menos petróleo extraíble en la corteza terrestre del que había entonces, ya no preocupa tanto su escasez como el "cambio climático" derivado del exceso de residuos. ¿A qué se debe tan llamativo cambio de percepción? Por una parte a que los países ricos mejoraron desde entonces su relación de intercambio, reduciendo notablemente la fracción de sus ingresos destinada al pago del petróleo y minimizando, así, la incidencia económica que sobre ellos tuvieron las recientes subidas de precios. Por otra parte, a que al ser los países ricos los principales consumidores de recursos, son los principales generadores de residuos y hacen de éstos su "primer problema ambiental" y tratan de exportarlo como tal al resto del mundo. ${ }^{1}$ Pero también a que el problema del agotamiento del petróleo se enfocó de forma muy simplista pronosticando crisis de abastecimiento para fechas tan perentorias que la realidad se encargó de desmentir, permitiendo tildar dichas previsiones de catastrofistas. En lo que sigue encajaremos el problema del petróleo en un enfoque más amplio y clarificador de la verdadera encrucijada a la que se enfrenta la civilización industrial.

Anticipemos que la especie humana moviliza hoy un tonelaje muy superior a la de cualquier fuerza geológica ${ }^{2}$ al articular mayoritariamente su intendencia sobre las extracciones de la corteza terrestre y no sobre los derivados renovables de la energía solar y la fotosíntesis. Esta potente actividad extractiva utiliza los minerales que la corteza terrestre

\footnotetext{
${ }^{1}$ Parece como si la contaminación cuyo control preocupa no procediera del desmesurado y despreocupado consumo de recursos. Además, dentro del problema de los residuos, se magnifica la contaminación atmosférica, causante tanto de cambios climáticos globales — que afectan a poblaciones enteras atendiendo más a su clima y ubicación geográfica que a su nivel de ingresos- como de trastornos locales más específicos de los países ricos, como es el caso de las lluvias ácidas, altamente destructivas en los suelos ácidos propios de clima húmedo templado pero no, por ejemplo, en los suelos calcáreos predominantes en clima mediterráneo.

${ }^{2}$ El transporte horizontal de materiales realizado por la erosión natural desde la cabecera de las cuencas hasta las zonas de sedimentación, se ha visto hoy ampliamente superado en tonelaje por el modelo que va desde las áreas de extracción hasta las grandes aglomeraciones, utilizando el viario y los medios de transporte de nueva factura. Véase Cendrero, A., Remondo, J. y Rivas, V. (2005) "Influencia humana en la evolución de los procesos superficiales" en Naredo, J.M. y Gutiérrez, L. (Eds.) (2005) La incidencia de la especie humana sobre la faz de la Tierra (1955-2005), Ed. Universidad de Granada y Fudación Césasr Marique, Col. "Ecomonía vs naturaleza", pp. 261-307.
} 
había concentrado en forma de yacimientos singulares que, tras ser utilizados, se dispersan originando normalmente problemas de contaminación. La metodología propuesta ${ }^{3}$ permite cuantificar globalmente el potencial que alberga el "capital mineral" de la Tierra, que la actual civilización está dispersando ahora a tanta velocidad. De esta manera si, como se dice, la vida surgió y evolucionó en la Tierra a partir de una sopa primigenia, la especie humana la empuja ahora con fuerza hacia una especie de puré crepuscular, cuya composición química hemos definido en las investigaciones indicadas como estado tendencial referencia. La metodología propuesta permite cuantificar la pérdida de potencial que va acusando el "capital mineral" de la Tierra a medida que la incidencia humana la empuja hacia esa especie de sopa entrópica o "estado muerto" de referencia, otorgando a las actuales preocupaciones por la "(in)sostenibilidad", dimensiones claramente cuantitativas que el ambientalismo banal de nuestros días trata de soslayar.

\section{“Capital natural” y capital reproductible}

Se ha dicho que el objetivo de sostenibilidad para un economista ha de pasar por una revalorización del "capital natural" que facilite su mantenimiento e incluso su mejora, incluyendo dicho patrimonio en la categoría de capital en general. El problema estriba en que, si bien el cálculo del coste físico y monetario de los bienes de capital producidos por la industria humana puede realizarse por procedimientos generalmente aceptados, no ocurre lo mismo con el "capital natural". En primer lugar, normalmente estos recursos no se identifican con valores monetarios. Y en segundo lugar, su reproducción, aún cuando resulte técnicamente viable, suele arrojar costes físicos y monetarios tan elevados que la hacen económicamente inviable. De ahí que se estime escasamente operativo el afán de cifrar la sostenibilidad ecológica de los sistemas económicos en el requisito de que su "capital natural" no disminuya o se degrade físicamente.

El enfoque propuesto en Naredo y Valero (dirs.) (1999) permite calcular, a partir de un estado de referencia, el coste físico de reposición de los recursos minerales de la corteza terrestre, acercando así por vez primera el tratamiento económico de esta categoría de recursos a la del capital reproductible. Con este enfoque se ofrecen nuevos criterios para trascender un grave escollo con el que se topa el análisis económico en el campo de los recursos naturales: el que plantea el hecho de que el análisis económico ordinario valore los stocks de recursos que nos ofrece la naturaleza atendiendo a su coste monetario de extracción (y manejo) y no al que exigiría su reposición. Con lo que esa valoración sesgada

\footnotetext{
${ }^{3}$ Esta propuesta vio la luz en Naredo, J.M. y Valero, A. (Dirs.) (1999) Desarrollo económico y deterioro ecológico, Fundación Argentaria y Visor Distribuciones, Col. "Economía y naturaleza", y ha sido desarrollada en tesis y publicaciones posteriores: véase, por ejemplo, Valero, A., Botero, E. y Martínez, A. (2005) "Evolucion y perspectivas del uso de la energía y los materiales" en Naredo, J.M. y Gutiérrez, L. (Eds.), Op. Cit., pp. 307-342.
} 
ha primado sistemáticamente la extracción frente a la recuperación y reciclaje (donde los costes de reposición se han de sufragar íntegramente). Este proceder acentúa tanto los problemas de escasez de recursos como los de exceso de residuos, a medida que el modelo de comportamiento propio de la civilización industrial se extiende y distancia cada vez más de aquel otro de la biosfera, que se caracteriza por cerrar los ciclos de materiales, convirtiendo, con la ayuda de la energía solar, los residuos en recursos. De esta manera, calcular en toda su globalidad los costes físicos (es decir, incluyendo el coste de reposición de los recursos naturales), en los que incurren los procesos productivos propios de la civilización industrial, parece un paso obligado para enjuiciar económicamente y para manejar con conocimiento de causa los instrumentos que inciden sobre la valoración, a fin de reorientarlos hacia una mayor sostenibilidad global. Siendo la estimación del coste físico de reposición de los recursos minerales el primer paso para hacer que la analogía entre el "capital natural" y el fabricado por el ser humano sea algo más que una metáfora vacía de contenido concreto.

\section{Extracción mineral o aprovechamiento de la fotosíntesis}

La propuesta metodológica aquí presentada se refiere al "capital mineral" y no al conjunto del "capital natural". Es decir, se refiere sólo a un subconjunto de elementos que componen ese "capital natural", dejando de lado el resto de los elementos y sistemas que se engloban también bajo esa denominación, entre los que destacan los integrantes de la biosfera, cuyo tratamiento demandaría planteamientos metodológicos distintos. Pese a esta limitación, el trabajo tiene gran relevancia al ser precisamente un rasgo distintivo de la civilización industrial el apoyar básicamente su intendencia en la extracción de rocas y minerales de la corteza terrestre en vez de hacerlo en los derivados de la fotosíntesis, como hacen el resto de las especies de la biosfera y como había hecho la especie humana a lo largo de su historia.

La civilización industrial se ha caracterizado, y sigue caracterizándose, por utilizar masivamente como materias primas determinadas sustancias disponibles en la corteza terrestre en condiciones muy particulares de concentración, estructura y tonelaje. Los yacimientos minerales en explotación pueden considerarse, así, como rarezas de la corteza terrestre, ya que cuentan con unas leyes de contenido en las sustancias deseadas y un nivel de estructura muy superiores a la media de la corteza terrestre, que la naturaleza se había encargado espontáneamente de configurar. Una vez utilizados estos recursos, suelen acabar dispersándose y originando los problemas de contaminación de todos conocidos, habida cuenta de que, las prácticas habituales de cálculo que orientan la gestión económica no acostumbran a favorecer la recuperación y el reciclaje. Y al tomar estos recursos como un don gratuito de la naturaleza se incentiva su extracción, no sólo frente a la recuperación y el reciclaje, sino también frente a otros posibles sustitutivos renovables fruto de la industria 
humana, que habría que producir y también facturar. ${ }^{4}$

El enfoque aquí utilizado permite ordenar económicamente los minerales de la corteza terrestre atendiendo al coste físico que supondría obtenerlos a partir de los materiales que contendría la Tierra si hubiera alcanzado ya el máximo nivel de deterioro hacia el que la estamos empujando (es decir, si los actuales yacimientos de rocas y minerales se hubieran mezclado y reaccionado con el resto de los componentes hasta formar una "sopa entrópica" en equilibrio químico). Expresando en unidades de energía el coste físico de obtener a partir de esa "sopa entrópica" los minerales hoy disponibles, podríamos llegar a calcular el potencial (stock) contenido en la corteza terrestre actual, que la especie humana puede explotar y dispersar con mayor o menor celeridad, por contraposición al flujo de energía emitido por el Sol y sus derivados renovables.

\section{Cerrar los ciclos de materiales. Coste de reposición y coste de extracción}

En consecuencia, la sostenibilidad o viabilidad ecológica de un sistema económico debe enjuiciarse atendiendo no tanto a la intensidad en el uso que hace de los stocks de recursos no renovables como a su capacidad para cerrar los ciclos de materiales mediante la recuperación o el reciclaje, con ayuda de fuentes renovables.

Recurriendo a la metodología derivada de este enfoque y aplicándola a los minerales contenidos en los yacimientos con leyes comerciales, podríamos extender el cálculo del coste exergético de concentración al conjunto de los recursos minerales contenidos en la corteza terrestre. Para ello habría que definir la forma en la que se distribuye actualmente el stock de cada sustancia en la corteza terrestre, relacionando las leyes de concentración y el tonelaje en los minerales que la contienen. La experiencia indica que la relación entre la ley (que podemos representar en el eje de ordenadas) y el tonelaje (en el de abcisas) adopta la expresión geométrica de funciones con pendiente negativa $\mathrm{y}$, generalmente, de exponenciales convexas hacia el origen de coordenadas, en las que la pendiente varía atendiendo a la situación peculiar de cada sustancia concreta. El hierro ejemplificaría el caso de sustancias muy abundantes y extendidas en la corteza terrestre. Esta sustancia mantiene una ley que apenas baja del $1 \%$ en casi toda la corteza terrestre, apareciendo buena parte del tonelaje con leyes iguales o ligeramente superiores a este porcentaje. Sin embargo en sustancias más raras y peor distribuidas, como el mercurio, el oro o el petróleo,

\footnotetext{
${ }^{4}$ Por ejemplo, se incentivó no sólo a sustituir la mula por el tractor, sino a que este funcionara con petróleo y no a partir de fuentes renovables (como ocurría en la postguerra, cuando automóviles y tractores circulaban utilizando el gasógeno para generar "gas pobre" mediante una combustión incompleta de residuos o biomasas poco valoradas, explotando ya lo que ahora se denominan "biocombustibles de segunda generación").
} 
el tonelaje se concentrará más en ciertos yacimientos. La construcción de este tipo de curvas, modelizando la relación entre leyes y tonelaje para las principales sustancias de la corteza terrestre que están siendo objeto de explotación, constituye una tarea pendiente para completar la aplicación de la metodología propuesta. ${ }^{5}$ Esta tarea es importante para romper el actual vacío que se observa entre la investigación geológica y la minera, que dificulta la modelización y clasificación de los yacimientos por escalones de coste físico y monetario de extracción.

Una vez modelizada la presencia de las sustancias en la corteza terrestre, podríamos aplicarle la metodología de cálculo del coste físico de reposición de dichas sustancias y, mediante agregación, cuantificar en unidades energéticas el coste físico que nos ahorra su extracción, al evitar tener que concentrarlas. Este coste sería indicativo del potencial que nos brinda la corteza terrestre en forma de concentración y enlace químico de sustancias minerales explotables. Lo cual permite plantear en términos meridianamente cuantitativos el conflicto fáustico en el que se debate la civilización industrial, al haber optado por utilizar la eficacia inmediata que le ofrece la simple explotación (y deterioro) de este potencial, aunque ello sea a costa de renunciar, de hecho, a la sostenibilidad global del sistema. La preocupación actual de hacer que el sistema atienda al objetivo de la sostenibilidad global, sin menoscabo de su eficiencia inmediata, resulta bastante problemático. Ya que tal objetivo exigiría que la sociedad se apoyara en la energía solar y sus derivados para restituir ese potencial reconvirtiendo los residuos en recursos, lo que nos llevaría a reconsiderar todos los procesos y a descartar muchos de ellos por incapaces o ineficientes a la hora de atender este requisito. Las centrales nucleares al uso serían un ejemplo de procesos a descartar, por no existir procedimiento técnico alguno que permita reconvertir en este caso los residuos en recursos, dejando así permanentemente abierto el ciclo de materiales. A la vez que el uso del petróleo como combustible debería ser descartado por ineficiente o impracticable si se pretendieran reconvertir los residuos en recursos: reconstruir los enlaces químicos de los hidrocarburos una vez quemados exigiría del orden de mil veces más energía de la liberada en su combustión. Así la única manera viable de reponerlos sería hacerlo indirectamente, a base de plantar y acumular los bosques necesarios para ello, pero esto exigiría un uso tan masivo de suelo y de tiempo, que imposibilitaría tal reposición a los ritmos a los que actualmente se están quemando. La

\footnotetext{
${ }^{5}$ Una metodología de este tipo ha sido diseñada y aplicada a los yacimientos españoles de estaño y wolframio, situándolos en el contexto mundial: Ortiz, A. (1993): "Los recursos mineros", en J.M.Naredo y F. Parra, editores, Hacia una ciencia de los recursos naturales, Madrid, Siglo XXI Editores. La investigación que está desarrollando Alicia Valero como tesis doctoral en la Universidad de Zaragoza, promete avances significativos en este y otros puntos de la metodología aquí esbozada. La tesis doctoral de Alicia Valero (2008) Enegy Evolution of the Mineral Capital on Earth (dirigida por Antonio Valero y presentada en la Universidad de Zaragoza) aporta avances significativos en la aplicación de nuestra metodología a la composición, distribución y evolución del capital mineral de la Tierra, cuantificado como un depósito de exergía que cabe utilizar y disipar más o menos rápidamente, en comparación con el flujo solar y otras fuentes renovables.
} 
exigencia de cerrar los ciclos de materiales reconvirtiendo los residuos en recursos, a fin de imitar el funcionamiento de la biosfera como ejemplo modélico de sistema de gestión y uso sostenible de los recursos planetarios, desbancaría a los combustibles fósiles del pedestal de eficiencia y competitividad en el que los sitúan los enfoques parcelarios al uso frente a las fuentes renovables de energía. El enfoque más amplio desde el que estamos razonando aconseja a distinguir, así, los procesos en los que no se conoce procedimiento alguno de hacerlos reversibles, de aquellos otros en los que existen pero no resultan practicables a la escala a la que se requiere, y, por último, aquellos en los que existiendo procedimientos y siendo teóricamente practicables en gran escala, merece la pena analizar sus costes físicos y monetarios a fin de estudiar su viabilidad técnica y económica con vistas a su posible implantación.

\section{El crecimiento exponencial, fenómeno transitorio en la historia humana}

Retomando el hilo de razonamiento anterior, hemos de advertir que la metodología que estamos proponiendo permite dar un salto cualitativo en los análisis que, desde el ángulo de la sostenibilidad global, se han venido aplicando al uso del "capital mineral" de la Tierra en la sociedad actual, deshaciendo algunos de los equívocos más comunes. Estos análisis han venido subrayando que la condición de stocks de los depósitos minerales en explotación hace insostenible el futuro de una sociedad que se asiente en la extracción y dispersión continuada de los mismos. Los trabajos de M. King Hubbert (1969 y 1974, reed. 1993) ${ }^{6}$ ocupan entre ellos un lugar importante, al aunar tempranamente la formalización matemática a la claridad expositiva. Tras constatar que estamos habituados a que una suma de dinero, que no tiene naturaleza física, se atenga a la regla del interés compuesto y aumente siguiendo indefinidamente una curva de crecimiento exponencial, este autor observa que la civilización industrial ha proyectado también sobre el mundo físico ese hábito del crecimiento exponencial, originando un comportamiento sin precedentes en la historia de la humanidad, que a largo plazo se muestra inviable: la novedad e inviabilidad de ese comportamiento se derivan de que $1^{\circ}$ ) se apoya masivamente sobre la extracción de recursos no renovables de la corteza terrestre y $2^{\circ}$ ) que practica estas extracciones de modo exponencial en el tiempo. Hubbert muestra con lápiz y papel que "ninguna cantidad física

\footnotetext{
${ }^{6}$ Hubbert, M.K. (1969): capítulo sobre recursos energéticos, en Committee on Resources and Man, National Academy of Sciences, National Research Council, Resources and Man, San Francisco, W.H.Freeman. Véase igualmente el trabajo de síntesis posterior: Hubbert, M.K. (1974) "Exponential Growth as a Transient Phenomenon in Human History”, en Strom M.A. (ed.) Societal Issues, Scientific Viewpoints, Nueva York, American Institute of Physics (Reed. en Daly. H.E. y Tawnsend, K.N. (eds.) (1993): Valuing the Earth: economics, Ecology, Ethics, Cambridge, Massachusetts, The MIT Press).
} 
puede seguir una curva exponencial más que durante un relativamente breve período de tiempo". Para ello pone en conocimiento de los legos en la materia las implicaciones matemáticas del crecimiento exponencial, mostrando con ejemplos que la persistencia en el tiempo del aumento de una población o la expansión de cualquier objeto físico a una tasa acumulativa constante se revela inviable a plazos relativamente cortos, al desatar crecimientos exponenciales que trascienden de las dimensiones nuestro planeta. De ahí que el autor mencionado presente el crecimiento exponencial, como explícitamente indica el título del segundo de sus trabajos citados, como "un fenómeno transitorio" en la historia de la humanidad. Y viendo que la explotación de determinados stocks de minerales, y muy particularmente los "energéticos", están siguiendo curvas exponenciales de crecimiento en nuestra actual civilización, se postula que tarde o temprano sus tasas de crecimiento tendrán que flexionar primero para transmutarse después en tasas de decrecimiento, dado el carácter limitado de estos stocks. Este autor enfrenta las curvas de extracción de determinados recursos minerales a la estimación de los stocks o reservas disponibles, utilizando para ello un procedimiento formalizado relativamente sencillo que facilita su interpretación. Veamos cómo se aplica al caso del petróleo.

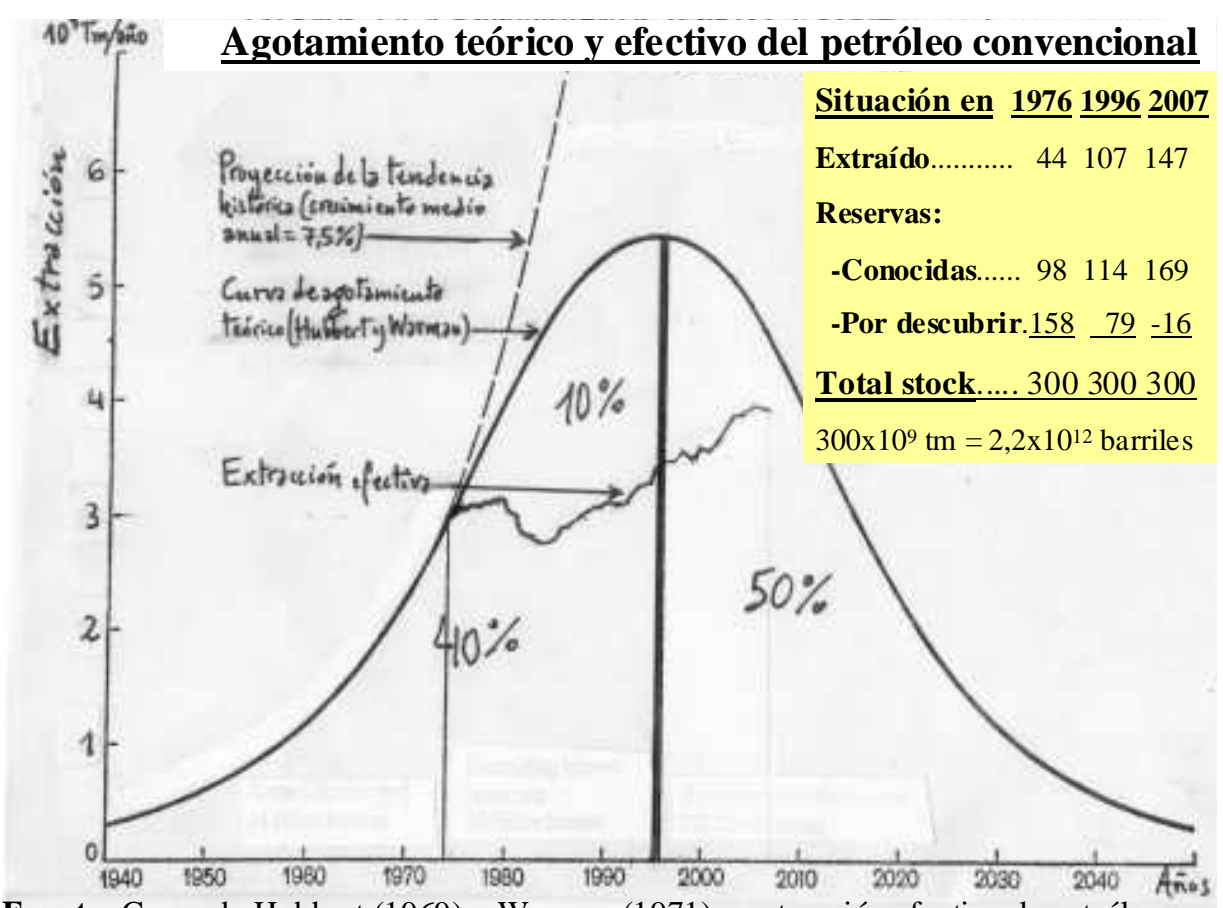

Fuente: Curva de Hubbert (1969) y Warman (1971) y extracción efectiva de petróleo.

El gráfico representa la extracción de petróleo en miles de millones de toneladas anuales, en el eje de ordenadas, y el tiempo en años, en el de abscisas. Este gráfico reproduce el ajuste que propusieron Hubbert y Warman, de una exponencial a la curva de 
extracciones tal y como se venía produciendo hasta la primera "crisis petrolera" de los setenta. También recoge la curva de agotamiento teórico, en que la superficie comprendida entre la curva y el eje de abscisas representa la estimación del stock de petróleo convencional cifrado en 300 mil millones de toneladas (equivalente a unos 2,2 billones [2,2 $\mathrm{x} 10^{12}$ ] de barriles). ${ }^{7} \mathrm{Al}$ atenerse la extracción al carácter limitado de la reserva, la pendiente de esta curva de explotación inflexiona desde la exponencial originaria hacia el decrecimiento, mostrando una de las posibles sendas que puede seguir en el tiempo la extracción de un recurso no renovable. Esta curva de explotación teórica preveía para principios de los setenta una inflexión hacia tasas de crecimiento cada vez menores que se prolongaría hasta alcanzar un máximo hacia 1996, a partir del cual empezaría a decrecer (dada la simetría de la curva este máximo coincide con el momento en el que se ha extraído la mitad del stock). Al añadir en el gráfico las toneladas de petróleo realmente extraídas hasta 1996, podemos observar que la inflexión producida tras la llamada crisis energética de los setenta fue bastante más acusada que la prevista en la curva de extracción teórica, aunque después volviera a repuntar, pero con tasas de crecimiento menores. De esta manera en 1996 se habría extraído el $40 \%$ del stock, en vez del $50 \%$ que preveía la curva (quedando un $60 \%$ por explotar). Por lo que habría que desplazar la curva de previsiones hacia la derecha (desplazando la fase de declive unos quince o veinte años, cosa que no hemos hecho en el gráfico por evitar hacerlo más farragoso, sobre todo cuando nuestro propósito no es actualizar este tipo de previsiones, sino discutir su interés y sus limitaciones, en relación con nuestra propuesta metodológica. En cualquier caso, al prolongar la curva de crecimiento de la extracción efectiva de petróleo hasta el presente, nos encontramos con que en 2008 viene a producirse el llamado "pico de Hubbert", que marcaría el punto en el que, al haberse extraído ya la mitad del petróleo convencional, la curva teórica de extracción ajustada en forma de campana de Gauss empezaría a decrecer. Pero este decrecimiento no tiene por que ser real. En primer lugar, hay experiencias en las que la explotación de sustancias minerales concretas ha seguido aumentado después de haber extraído ya la mitad de las reservas, para caer más tarde y con mayor brusquedad de lo que señalaba a curva de Hubbert. En segundo lugar, pueden aparecer más reservas de las que estaban previstas: los datos del cuadro incluidos en el gráfico muestra que así ha debido ser, cuando al poner los datos de consumo y de reservas probadas (tomadas del boletín estadístico de British Petroleum), suman ya más del los 300 mil millones de toneladas de reservas totales que veníamos considerando (poniendo en el cuadro reservas por descubrir negativas, para subrayar esta incoherencia estadística). En tercer lugar, puede seguir aumentando el consumo de petróleo, a la vez que disminuye la extracción de petróleo convencional, al aumentar la fracción extraída de petróleo no convencional, o de petróleo obtenido a partir del carbón o de distintas biomasas o residuos a partir de la reacción Fisher-Tropps, utilizada ya a escala industrial para abastecer el grueso del ejército alemán durante la segunda guerra mundial. Pero, en cuarto lugar, lo que sigue gobernando el

\footnotetext{
${ }^{7}$ Una tonelada de crudo $=7,3$ barriles.
} 
consumo de petróleo y el uso de otras fuentes de energía es el pulso de la coyuntura económica, por lo que la crisis actual provocará sin duda otro significativo diente se sierra en la curva de extracción efectiva, comparable con el observado tras las crisis petrolíferas de los setenta, que marcaron el inicio de la sustitución de esta fuente de energía. También la caída del precio del petróleo -que actualmente viene a ser un tercio de los precios máximo alcanzados hace solo unos meses- denota que este depende más de los episodios a corto plazo de la coyuntura económica, que del nivel de reservas de la corteza terrestre.

Hemos de subrayar que si hemos tomado el petróleo convencional como base de este ejercicio es, entre otras cosas, porque es uno de los recursos mineros mejor estudiados en la corteza terrestre. Esto hace que la estimación en torno a los dos billones de barriles de reservas se haya mantenido vigente como una cifra razonable a lo largo de los últimos veinte años. Fue entre la década de los treinta y la de los sesenta cuando se descubrieron las dos decenas de depósitos "supergigantes" de Oriente Medio que acumulaban cerca de la tercera parte de la reservas mundiales. Hoy se estiman descubiertos al menos el $80 \%$ de los estratos sedimentarios existentes susceptibles de contener petróleo, y ha sido explorada la práctica totalidad de los accesibles a bajo coste, por lo que al parecer no caben grandes sorpresas en este campo. Lo cual reduce la incidencia de una primera limitación al uso del tipo de ejercicios matemáticos propuestos por Hubbert: la que se deriva del desconocimiento de las reservas disponibles, que hace que la curva de agotamiento se vaya desplazando hacia la derecha debido a los nuevos descubrimientos, aunque la extracción siga creciendo exponencialmente. Todo ello ante la desconexión antes mencionada entre las estimaciones de la geología sobre la composición de la corteza terrestre y la investigación minera en su búsqueda de esas rarezas que en dicha corteza son los yacimientos.

Insistamos que una segunda limitación de este tipo de análisis procede de la definición imprecisa del recurso cuyas reservas se pretenden estimar y relacionar con las extracciones. Por ejemplo no tiene mucho sentido hablar de agotamiento del hierro, cuando se trata de una sustancia muy abundante en la corteza terrestre que por mucho que se oxide y disperse seguirá siendo abundante. Pero sí tendría sentido hablar del agotamiento de determinados yacimientos con ciertas características de ley, tonelaje y accesibilidad, que corresponderían a ciertos tramos de coste físico y monetario de extracción. Aunque para ello habría que avanzar -como ya se ha indicado- en la modelización de la composición y distribución de las sustancias de la corteza terrestre, y de los yacimientos que contiene. En el caso del petróleo que estamos utilizando como ejemplo, habría que distinguir entre las reservas en esos depósitos "supergigantes" y fácilmente accesibles del Oriente Medio u otros cuyo coste no exceda de los $10 \$$ el barril, de los yacimientos más pequeños, situados a mayor profundidad, en el mar, etc, que requieren procedimientos de extracción más onerosos, cuyos costes de extracción se sitúan entre los 10 y $20 \$$ el barril. A este "petróleo convencional caro", que empezó a explotarse tras las "crisis petroleras" de los setenta, le 
seguirían otros de mayor coste todavía entre los que cabe esperar se encuentre el grueso de las reservas cuya puesta en explotación todavía no se ha producido: se trata del "nuevo petróleo", que daría paso al "petróleo no convencional", cuya extracción y proceso mostraría unos costes mucho más elevados.

Por otra parte, no está de más recordar que ya durante la segunda guerra mundial el ejército alemán pudo paliar la penuria de petróleo convencional recurriendo al petróleo obtenido por la industria química a partir del carbón, al igual que en Brasil se ha estado sustituyendo por etanol obtenido a partir de biomasas. Por lo que incluso en un recurso aparentemente tan claro como el petróleo, las reservas no plantean tanto un problema de cantidad como de costes. El verdadero problema estriba en que la era del petróleo barato parece estar aproximándose a su fin. A un nivel más general, cuando en el $\mathrm{CERN}^{8}$ se consiguió hacer realidad la vieja quimera de "transmutar" plomo en oro, pero a un coste tan elevado que despojaba la operación de todo interés económico, podemos decir que el problema que plantea el abastecimiento de los llamados recursos naturales no renovables es cada vez más un problema de costes y no de imposibilidad física de obtenerlos, lo cual revaloriza nuestra propuesta metodológica orientada precisamente al cálculo de los costes físicos por procedimientos susceptibles de aplicación general y agregación.

Concluyendo: para que el esquema analítico de Hubbert adquiera plena utilidad, ha de aplicarse a dos niveles. Uno, micro, referido a depósitos minerales concretos inequívocamente identificados y otro el de máxima agregación, referido al conjunto de la corteza terrestre, que es la que en última instancia marca los stocks de forma también inequívoca.

Los trabajos de Campbell ${ }^{9}$ ofrecen un buen ejemplo de análisis desagregado, en el que se desglosan las reservas de petróleo por yacimientos y países, indicando en cada caso si la curva de agotamiento ha superado la mitad del stock e iniciado la fase de declive. Este análisis pormenorizado es el que permite agregar después por grupos y obtener conclusiones más solventes (aunque dependan siempre, claro está, de la validez de los datos disponibles, que a veces aparecen alterados por razones políticas y comerciales). Este autor completa, por último, su análisis del petróleo "convencional", con el del gas y el petróleo "no convencional", advirtiendo cómo el primero empezó a cobrar peso a partir de la década de los setenta y el segundo lo hará en los primeros años del nuevo milenio, sin que ambos consigan evitar el declive del conjunto de los hidrocarburos extraídos que se prevé para este siglo que comienza. La tesis de Alicia Valero antes citada ofrece un buen ejemplo de la aplicación de la Curva de Hubbert para diversas sustancias minerales a escala planetaria,

\footnotetext{
${ }^{8}$ Centro de investigación sobre altas energías con sede en Ginebra.

${ }^{9}$ Campbell, C.J. (1997:) "Depletion patterns show change due for production of conventional oil", Oil \& Gas Journal, 29 de diciembre 1997.
} 
tras haber elaborado la metodología que permite expresar esas sustancias en unidades de energía. Como consecuencia de ello muestra que el "pico de Hubbert" del gas se produciría en el año 2023, el del carbón en 2060, el del aluminio en 2057, el del hierro en 2068...

\section{Stock no renovable y flujo solar de exergía}

Pero la sustitución que se prevé para los hidrocarburos se ha producido y se seguirá produciendo entre las sustancias que componen la corteza terrestre en general. Por lo que un mínimo afán de globalidad y coherencia exigiría referir los análisis de sustancias concretas al agregado último de la corteza terrestre, siguiendo los pasos iniciados en este trabajo. Para ello lo verdaderamente significativo no son las cantidades de tales sustancias sino la energía de calidad o exergía de concentración y enlace químico de los minerales que las contienen, que nos ofrece la corteza terrestre en forma de yacimientos. Exergía que hemos calculado para algunas sustancias, ${ }^{10}$ dando las orientaciones necesarias para generalizar y agregar dicho cálculo para el conjunto de la corteza terrestre, abarcando así desde una perspectiva más amplia el problema que la sustitución de materias primas que escapa al estudio de cada sustancia por separado, limitando su interés. De esta manera podríamos llegar a representar la curva de agotamiento del potencial de la corteza terrestre en un esquema como el presentado para el petróleo en el gráfico adjunto. Lo cual nos permitiría comparar este stock de potencia, con el flujo de exergía que nos envía diariamente en Sol y sus posibles derivados. Al igual que en su día se comparó la exergía en combustión que podría liberar el stock de combustibles fósiles, con la de origen solar (viendo que la almacenada por el petróleo equivalía a unos cinco días de radiación solar y la del total de combustibles fósiles a quince ${ }^{11}$ ), la aplicación de la metodología propuesta permitiría hacerlo para el conjunto de la exergía almacenada en la corteza terrestre, expresando en términos meridianamente cuantitativos el conflicto que plantea en términos físicos la sostenibilidad global de la civilización que nos ha tocado vivir. Con todo, hemos de advertir que no consideramos que el enfoque propuesto deba ser el único de interés para orientar la gestión del "capital mineral" de la Tierra. Pero sí el más importante para cuantificar en términos de coste físico (no de valor) la contribución de los recursos minerales al proceso económico y enjuiciar su sostenibilidad. Lo cual supone, nada más y nada menos, un prerrequisito esencial para enjuiciar la gestión sobre las bases racionales que ofrece la ciencia cuantitativa. Pero no permite cerrar el tema de la gestión proponiendo este o aquel óptimo. Es más, estimamos que cualesquiera que sean las decisiones que la

\footnotetext{
${ }^{10}$ J.M Naredo y A. Valero, dirs. (1999), op. cit., así como la tesis de de Alicia Valero antes citada.

${ }^{11}$ Georgescu-Roegen, N. (1972) "Energy and Economic Myths", reed. (1976) en Energy and Economic Myths and Institucional and Analytical Economic Essays, Nueva York, Pergamon Press (publicado en castellano en El Trimestre Económico, octubre-diciembre, 1975).
} 
REVISTA POLIS

VOL. $7 \mathrm{~N}^{\circ} 21,2008$

PRÒLOGO, págs., 91-104

○ EDITORIAL DE LA UNIVERSIDAD BOLIVARIANA DE CHILE, 2008

gestión adopte, serán fruto de juicios de valor, inclinaciones e instituciones, cuyas orientaciones escapan necesariamente al campo de la ciencia cuantitativa, aunque puedan venir informadas por ésta. 


\section{Bibliografía}

Campbell, C.J. (1997:) "Depletion patterns show change due for production of conventional oil", Oil \& Gas Journal, 29 de diciembre 1997.

Cendrero, A., Remondo, J. y Rivas, V. (2005), "Influencia humana en la evolución de los procesos superficiales" en Naredo, J.M. y Gutiérrez, L. (eds.) La incidencia de la especie humana sobre la faz de la Tierra (1955-2005), Ed. Universidad de Granada y Fundación César Manrique, Lanzarote.

Daly, H.E; Naredo, J.M. y Valero, A. (dirs.) (1999), Desarrollo económico y deterioro ecológico, Fundación Argentaria y Visor Distribuciones, Col. "Economía y naturaleza", Madrid.

Georgescu-Roegen, N. (1972), "Energy and Economic Myths", reed. (1976), en Energy and Economic Myths and Institucional and Analytical Economic Essays, Pergamon Press, Nueva York (publicado en castellano en El Trimestre Económico, octubre-diciembre, 1975).

Hubbert, M.K. (1969), capítulo sobre recursos energéticos, en Committee on Resources and Man, National Academy of Sciences, National Research Council, Resources and Man, W.H.Freeman, San Francisco.

Hubbert, M.K. (1974), "Exponential Growth as a Transient Phenomenon in Human History”, en Strom M.A. (ed.) Societal Issues, Scientific Viewpoints, American Institute of Physics, Nueva York .

Naredo, J.M. y Gutiérrez, L. (eds.) (2005), La incidencia de la especie humana sobre la faz de la Tierra (19552005), Ed. Universidad de Granada y Fundación César Manrique, Lanzarote.

Ortiz, A. (1993), "Los recursos mineros", en Naredo, J.M.y Parra, F. (eds.) Hacia una ciencia de los recursos naturales, Siglo XXI Editores, Madrid.

Sempere J. y Tello, E. (coords.) (2007) El final de la era del petróleo barato, Icaria, Barcelona.

Tawnsend, K.N. (eds.) (1993), Valuing the Earth: economics, Ecology, Ethics, The MIT Press, Cambridge, Massachusetts.

Valero, A., Botero, E. y Martínez, A. (2005), "Evolución y perspectivas del uso de la energía y los materiales" en Naredo, J.M. y Gutiérrez, L. (eds.), La incidencia de la especie humana sobre la faz de la Tierra (1955-2005), Ed. Universidad de Granada y Fundación César Manrique, Lanzarote. 